\title{
CHARACTERIZATIONS OF METRIC PROJECTIONS IN BANACH SPACES AND APPLICATIONS
}

\author{
JEAN-PAUL PENOT AND ROBERT RATSIMAHALO
}

\begin{abstract}
This paper is devoted to the study of the metric projection onto a nonempty closed convex subset of a general Banach space. Thanks to a systematic use of semi-inner products and duality mappings, characterizations of the metric projection are given. Applications to decompositions of Banach spaces along convex cones and variational inequalities are presented.
\end{abstract}

\section{INTRODUCTION}

A number of problems can be reformulated as best approximation problems in some normed vector spaces. In several instances, the choice of the norm is imposed by the nature of the problem, so that one does not always dispose of an Hilbertian structure. It is the purpose of the present paper to describe characterizations of the solutions (which are not necessarily unique) of such problems in case the feasible set is convex. Such characterizations are known under restrictive assumptions such as Gâteaux-differentiability of the norm off 0 , or strict convexity of the norm (see [16], [22], [2], [3]). Here, we dispense with such assumptions by making a strong use of the concepts of duality mapping and of semi-inner product. These notions are recalled in section 2. Section 3 presents the characterization we have in view. In sections 4 and 5, some applications in the field of ordered Banach spaces and variational inequalities are presented. Applications to sensitivity analysis will be considered elsewhere. We thank Professor A Seeger for providing us with reference [32]. We are grateful to Professor Y. Al'ber for a careful reading of our manuscript which led us to improve its presentation and for pointing out to us several references on projections and generalized projections which complete our bibliography ([1], [3], [4]), in particular reference

1991 Mathematics Subject Classification. 90C48.

Key words and phrases. Metric projection, closed convex subset of a Banach space, semi-inner products, duality mappings, convex cones.

Received: May 15, 1997. 
[2] which is related to Section 5 of the present paper. We are also thankful to Professor C. Zalinescu for calling our attention on the formulation of Proposition 5.1 of the present paper.

\section{Preliminaries}

In the sequel, $X$ is a Banach space with topological dual $X^{*}$, and $\langle.,$. is the usual duality pairing between $X^{*}$ and $X$. We set $k(x)=\frac{1}{2}\|x\|_{X}^{2}$, $n(x)=\|x\|_{X}$, and we denote by $\mathcal{F}(X)$ (resp. $\left.\mathcal{C}(X)\right)$ the set of nonempty closed subsets (resp. closed convex subsets) of $X$. The duality mapping (or rather multimapping) $J$ is defined by

$$
J(x)=\left\{x^{*} \in X^{*}:\left\langle x^{*}, x\right\rangle=\|x\|^{2},\left\|x^{*}\right\|=\|x\|\right\} .
$$

It is monotone (see for example [11]), as it coincides with the subdifferential of the convex function $k$. Here we identify a multimapping with its graph:

$$
\left\langle x^{*}-y^{*}, x-y\right\rangle \geq 0, \forall\left(x, x^{*}\right),\left(y, y^{*}\right) \in J .
$$

The duality mapping $J$ is also homogeneous. As this correspondence will be of fundamental use in this paper, let us recall some of its properties. Since the function $k$ (resp. $n$ ) is convex, continuous at each point, its radial derivative exists. Using classical notations (see for example [17], [11], [24], $[22]$ ), we set:

$$
\begin{gathered}
\langle x, y\rangle_{+}:=k^{\prime}(x, y)=\lim _{t \rightarrow 0_{+}} \frac{\|x+t y\|^{2}-\|x\|^{2}}{2 t} \\
\text { (resp. } \left.[x, y]_{+}:=n^{\prime}(x, y)=\lim _{t \rightarrow 0_{+}} \frac{\|x+t y\|-\|x\|}{t}\right) .
\end{gathered}
$$

The bracket $\langle., .\rangle_{+}$(resp. $[., .]_{+}$) is called the semi-inner product (resp. the normalized semi-inner product) (see [17]). Moreover, the subdifferential $J=$ $\partial k$ (resp. $S=\partial n$ ) of $k$ (resp. of $n$ ) is related to the semi-inner product through the relation

$$
\begin{gathered}
\langle x, y\rangle_{+}=\max \left\{\left\langle x^{*}, y\right\rangle: x^{*} \in J(x)\right\} \\
\text { (resp. }[x, y]_{+}=\max \left\{\left\langle x^{*}, y\right\rangle: x^{*} \in S(x)\right\},
\end{gathered}
$$

(see for example [24], [11]), which is a special case of the relation

$$
f^{\prime}(x, y)=\max \left\{\left\langle x^{*}, y\right\rangle: x^{*} \in \partial f(x)\right\},
$$

for $f$ a continuous convex function. Thus, one sees easily that

$$
\left|\langle x, y\rangle_{+}\right| \leq\|x\|\|y\| \text { and }\langle x, y\rangle_{+}=\|x\|[x, y]_{+} .
$$

The map which sends $x$ to $\langle x, y\rangle_{+}$, for a given $y$, is positively homogeneous. The map which sends $y$ to $\langle x, y\rangle_{+}$(resp. $\left.[x, y]_{+}\right)$, for a given $x \neq 0$, is sublinear continuous. If $k$ is Gâteaux-differentiable, $J$ is single-valued, and $S$ is single-valued on $X \backslash\{0\}$. Also, $J(0)=\{0\}, S(0)=B_{X^{*}}$, and for $x \neq 0$,

$$
\langle x, y\rangle_{+}=\langle J(x), y\rangle=\left\langle k^{\prime}(x), y\right\rangle \text { and }[x, y]_{+}=\langle S(x), y\rangle=\left\langle n^{\prime}(x), y\right\rangle .
$$


Let us recall that the semi-inner product is symmetric if, and only if, the mapping $J$ is linear if, and only if, the space $X$ is Hilbertian (see [30]). In this case, it coincides with the inner product of $X$, and if the space $X$ is identified with its topological dual $X^{*}$, the mapping $J$ is the identity mapping on $X$. Now, let us give some examples of duality mappings which will be used later on.

Let $X=L^{p}(\Omega, \mathbb{R})$, with $1<p<+\infty$, where $(\Omega, \mathcal{A}, \mu)$ is a measured space. In this case, $X^{*} \approx L^{q}(\Omega, \mathbb{R})$ with $\frac{1}{p}+\frac{1}{q}=1$. One has for $x \neq 0$

$$
J(x)=\|x\|^{2-p}|x|^{p-1} \operatorname{sgn} x,
$$

Here $\|x\|=\left(\int_{\Omega}|x(s)|^{p} d \mu(s)\right)^{\frac{1}{p}}$, and $\operatorname{sgn} x=\mathbb{1}_{[x>0]}-\mathbb{1}_{[x<0]}$. Hence, one has

$$
\langle J(x), y\rangle=\langle x, y\rangle_{+}=\|x\|^{2-p} \int_{\Omega}|x(s)|^{p-2} x(s) y(s) d \mu(s) .
$$

If $p=1$, identifying $X^{*}$ with $L^{\infty}(\Omega, \mathbb{R})$, then for $x \neq 0, J(x)$ is the set of $y^{*}=\|x\| x^{*}$ where $x^{*}$ satisfies $x^{*}=1$ on $[x>0], x^{*}=-1$ on $[x<0]$, $\left|x^{*}\right| \leq 1$ on $[x=0]$ (see for example [10], [31]). Thus, we obtain that $\langle x, y\rangle_{+}=\|x\|\left(\int_{[x>0]} y(s) d \mu(s)-\int_{[x<0]} y(s) d \mu(s)+\int_{[x=0]}|y(s)| d \mu(s)\right)$.

Example. Given a compact topological space $T$, let us consider $X=C(T)$, the space of continuous functions from $T$ into $\mathbb{R}$ equipped with the norm of uniform convergence, $\|x\|:=\sup \{|x(t)|: t \in T\}$. For $y \in X$, we set

$$
T(y)=\{t \in T:|y(t)|=\|y\|\} .
$$

It is also known (see [11]) that

$$
\langle x, y\rangle_{+}=\max \{x(t) y(t): t \in T(x)\} .
$$

Example. Let $\Omega$ be a bounded open subset of $\mathbb{R}^{d}$ and let $X=H_{0}^{1}(\Omega)$, the closure of $\mathcal{C}_{0}^{\infty}(\Omega)$ in $H^{1}(\Omega)$ for the norm induced by $H^{1}(\Omega)$ which is given by

$$
\|x\|_{H^{1}(\Omega)}=\left(\int_{\Omega}|x(t)|^{2} d \mu(t)+\sum_{i=1}^{d} \int_{\Omega}\left|\frac{\partial x(t)}{\partial t_{i}}\right|^{2} d \mu(t)\right)^{\frac{1}{2}} .
$$

If we choose on $X$ the norm $\|\cdot\|_{H^{1}(\Omega)}$ defined above, then, identifying $X=H_{0}^{1}(\Omega)$ with its topological dual $X^{*}=H^{-1}(\Omega)$, it is easily seen that $J$ is the identity mapping on $X$. However, let us consider the following norm on $X$

$$
\|x\|_{H_{0}^{1}(\Omega)}=\left(\sum_{i=1}^{d} \int_{\Omega}\left|\frac{\partial x(t)}{\partial t_{i}}\right|^{2} d \mu(t)\right)^{\frac{1}{2}},
$$

which is equivalent to the norm $\|\cdot\|_{H^{1}(\Omega)}$ (see for example [9]), since the open subset $\Omega$ is bounded. The square of this norm being differentiable, for all $x \in H_{0}^{1}(\Omega)$, there exists a unique $J(x) \in H^{-1}(\Omega)$ such that for all $y \in H_{0}^{1}(\Omega)$ :

$$
\langle J(x), y\rangle=\sum_{i=1}^{d} \int_{\Omega} \frac{\partial x(t)}{\partial t_{i}} \frac{\partial y(t)}{\partial t_{i}} d \mu(t) .
$$


It follows that

$$
J(x)=-\sum_{i=1}^{d} \frac{\partial^{2} x(t)}{\partial t_{i}^{2}}
$$

in the sense of distributions. Moreover, if $x \in H_{0}^{1}(\Omega) \cap H^{2}(\Omega), J(x)=-\triangle x$ in $L^{2}(\Omega)$, where $\triangle$ is the usual Laplacian calculated at $x$.

More properties of semi-inner products can be given thanks to the following extension to infinite dimensions of a result of Rockafellar (see [27]).

Proposition 2.1. Let $f$ be a convex function from $X$ into $\mathbb{R} \cup\{\infty\}$, finite and continuous on an open subset $U$ of $X$. The function $d f$ from $U \times X$ into $\mathbb{R}$ given by $d f(x, y)=f^{\prime}(x, y)$ is upper semicontinuous for the strong topology on $U \times X$. In particular, the map which sends $(x, y)$ to $\langle x, y\rangle_{+}$is upper semicontinuous for the strong topology. Moreover, if $f$ is Gâteauxdifferentiable on $U$, then $d f$ is continuous on $U \times X$ for the strong topology.

Proof. 1) Let $(x, y) \in U \times X$ and let $\left(x_{n}\right)_{n \in \mathbb{N}} \rightarrow x$ and $\left(y_{n}\right)_{n \in \mathbb{N}} \rightarrow y$. For all $r>f^{\prime}(x, y)$, there exists $t_{0}>0$ such that for all $\left.\left.t \in\right] 0, t_{0}\right]$ :

$$
r>t^{-1}(f(x+t y)-f(x)) .
$$

Since $f$ is continuous, there exists $n_{0} \in \mathbb{I N}$ so that for $n \geq n_{0}$

$$
r>t_{0}^{-1}\left(f\left(x_{n}+t_{0} y_{n}\right)-f\left(x_{n}\right)\right) .
$$

Hence for $n \geq n_{0}$ and $\left.\left.t \in\right] 0, t_{0}\right]$, one has, thanks to the convexity of $f$ :

$$
r>t^{-1}\left(f\left(x_{n}+t y_{n}\right)-f\left(x_{n}\right)\right) \geq f^{\prime}\left(x_{n}, y_{n}\right) .
$$

Thus $d f$ is upper semicontinuous on $U \times X$.

2) Suppose that $f$ is Gâteaux-differentiable at $x$. Then for each $y \in X$, $f^{\prime}(x, y)=-f^{\prime}(x,-y)$. Using the upper semicontinuity of $d f$,

$$
\lim \sup _{n \in \mathbb{N}} f^{\prime}\left(x_{n},-y_{n}\right) \leq f^{\prime}(x,-y)
$$

which may be rewritten, as $-f^{\prime}\left(x_{n},-y_{n}\right) \leq f^{\prime}\left(x_{n}, y_{n}\right)$,

$$
\lim \sup _{n \in \mathbb{N}}-f^{\prime}\left(x_{n}, y_{n}\right) \leq-f^{\prime}(x, y)
$$

It follows that

$$
\lim \inf _{n \in \mathbb{N}} f^{\prime}\left(x_{n}, y_{n}\right) \geq f^{\prime}(x, y) \geq \lim \sup _{n \in \mathbb{N}} f^{\prime}\left(x_{n}, y_{n}\right) .
$$

Thus $d f$ is continuous at $(x, y)$.

Under a stronger assumption, the conclusion of Proposition 2.1 can be reinforced as follows.

Proposition 2.2. Let $f$ be a convex function from $X$ into $\mathbb{R} \cup\{\infty\}$ finite and continuous on an open subset $U$ of $X$. If $f$ is Fréchet-differentiable at some $x \in U$, then for each $y \in Y$, the function $d f$ from $U \times X$ into $\mathbb{R}$ given by $d f(u, v)=f^{\prime}(u, v)$ is upper semicontinuous at $(x, y)$ for the product of the strong topology and the bounded weak topology. In particular, when $k$ is Fréchet-differentiable the map which sends $(x, y)$ to $\langle x, y\rangle_{+}$is upper 
semicontinuous for the product of the strong topology and the bounded weak topology.

Proof. Let $x \in U$ and let $\left(x_{i}\right)_{i \in I} \rightarrow x$ and $\left(y_{i}\right)_{i \in I} \rightarrow y$ weakly, with $\left(y_{i}\right)_{i \in I}$ bounded. Let $r\rangle\left\langle f^{\prime}(x), y\right\rangle$. For all $i$ and $x_{i}^{*} \in \partial f\left(x_{i}\right)$ such that $\left\langle x_{i}^{*}, y_{i}\right\rangle=$ $f^{\prime}\left(x_{i}, y_{i}\right)$ (such an $x_{i}^{*}$ exists by a result of Moreau), one has

$$
\left\langle x_{i}^{*}, y_{i}\right\rangle \leq\left\|x_{i}^{*}-f^{\prime}(x)\right\|\left\|y_{i}\right\|+\left\langle f^{\prime}(x), y_{i}-y\right\rangle+\left\langle f^{\prime}(x), y\right\rangle .
$$

Since the function $f$ is Fréchet-differentiable at $x,\left\|x_{i}^{*}-f^{\prime}(x)\right\| \rightarrow 0$ (see [5], [23]), hence for $i$ large enough $r>\left\langle x_{i}^{*}, y_{i}\right\rangle$; thus $d f$ is upper semicontinuous.

The following property of semi-inner products will be useful. (see [19], [29]).

Lemma 2.3. Let $w \in X$ and $x \in C$. The following assertions are equivalent:

(i) $\langle x-w, y-x\rangle_{+} \geq 0, \forall y \in C$.

(ii) $\langle y-w, y-x\rangle_{+} \geq 0, \forall y \in C$.

Proof. Assertion (i) implies (ii) because the multimapping $J$ is monotone, hence for all $u^{*} \in J(x-w)$ and all $v^{*} \in J(y-w),\left\langle u^{*}, y-x\right\rangle \geq\left\langle v^{*}, y-x\right\rangle$. It follows that

$$
\max \left\{\left\langle u^{*}, y-x\right\rangle: u^{*} \in J(x-w)\right\} \leq \max \left\{\left\langle v^{*}, y-x\right\rangle: v^{*} \in J(y-w)\right\},
$$

that is

$$
0 \leq\langle x-w, y-x\rangle_{+} \leq\langle y-w, y-x\rangle_{+} .
$$

Conversely, assertion (ii) implies (i). Indeed, by replacing $y$ by $t y+(1-t) x$ with $t \in] 0,1[$ in (ii), the upper semi-continuity of the semi-inner product enables us to write that

$$
\langle x-w, y-x\rangle_{+} \geq \lim \sup _{t \rightarrow 0_{+}} \frac{1}{t}\langle(1-t) x+t y-w, t(y-x)\rangle_{+} \geq 0 .
$$

\section{Characterizations of metric projections}

In this section we study the metric projection onto a nonempty closed convex subset $C$ of the Banach space $X$. The characterizations we present generalize those which are known in Euclidean and Hilbertian spaces. They are more general than the one obtained in [16] and [22], since no special geometrical property on the space is assumed.

For $w \in X, P(C, w)$ is the set of solutions to the programming problem:

$$
(\mathrm{P}): \min \left\{\frac{1}{2}\|x-w\|^{2}: x \in C\right\}=\min \{k(x-w): x \in C\} .
$$

It is also the set of minimizers of the distance function $d_{C}$ on $C$ given by

$$
d_{C}(w)=\inf \{\|x-w\|: x \in C\} .
$$


In the following, it is always assumed that the metric projection operator $P(C,$.$) is nonempty valued. Let us recall that for C \in \mathcal{F}(X)$, the (upper) tangent cone to $C$ at $x \in C$ is given by

$$
T(C, x)=\lim \sup _{t \rightarrow 0_{+}} t^{-1}(C-x) .
$$

When $C$ is convex, it is the closure of the radial tangent cone

$$
T^{r}(C, x)=\mathbb{R}_{+}(C-x) .
$$

The normal cone to $C$ at $x$ is the polar cone of the tangent cone:

$$
N(C, x)=(T(C, x))^{\circ}=\left\{x^{*} \in X^{*}:\left\langle x^{*}, x\right\rangle \leq 0 \forall x \in T(C, x)\right\} .
$$

For $C$ convex,

$$
N(C, x)=(T(C, x))^{\circ}=\left\{x^{*} \in X^{*}:\left\langle x^{*}, y-x\right\rangle \leq 0 \forall y \in C\right\} .
$$

Now, we are in a position to give fundamental characterizations of metric projections in general Banach spaces.

Theorem 3.1. For a nonempty closed convex subset $C$ of $X$ and $w \in X$, the following assertions are equivalent:

(a) $x \in C$ is a metric projection of $w$ in $C$, that is $x \in P(C, w)$;

(b) $0 \in \partial k(x-w)+N(C, x)$;

(c) $\exists v^{*} \in J(x-w):\left\langle v^{*}, v\right\rangle \geq 0 \forall v \in T(C, x)$;

(d) $\exists u^{*} \in J(w-x):\left\langle u^{*}, v\right\rangle \leq 0 \forall v \in T(C, x)$;

(e) $J(w-x) \cap N(C, x) \neq \emptyset$;

(f) $\langle x-w, v\rangle_{+} \geq 0, \quad \forall v \in T(C, x)$;

(g) $\langle x-w, y-x\rangle_{+} \geq 0, \quad \forall y \in C$;

(h) $\langle y-w, y-x\rangle_{+} \geq 0, \quad \forall y \in C$;

(i) $\langle w-x, x-y\rangle_{+} \geq 0, \quad \forall y \in C$;

(j) $\langle w-y, x-y\rangle_{+} \geq 0, \quad \forall y \in C$.

Proof. Let $i_{C}$ be the indicator function of $C$ given by $i_{C}(x)=0$ if $x \in$ $C, i_{C}(x)=+\infty$ otherwise. For a given $w \in X$, let $k_{w}$ be the convex function given by $k_{w}(x)=k(x-w)$. Since the function $k_{w}$ is finite, convex, continuous, usual subdifferential calculus rules ensure that

$$
0 \in \partial\left(k_{w}+i_{C}\right)(x)=\partial k_{w}(x)+\partial i_{C}(x)
$$

is a necessary and sufficient optimality condition for $x$ to be a minimizer of $k_{w}+i_{C}$ (see for example [27], [23] and, for recent monographs, [6], [13]). Since $\partial k_{w}(x)=\partial k(x-w), \partial i_{C}(x)=N(C, x)$, the equivalence of (a) and (b) follows. Condition (c) is obviously a reformulation of (b) as $\partial k(x-w)=$ $J(x-w)$. Condition (d) is seen to be equivalent to (c) by setting $v^{*}=-u^{*}$, $J$ being odd. Condition (e) is obviously a reformulation of (d) by the very definition of $N(C, x)$ as the polar cone of $T(C, x)$. Since $\langle x-w, v\rangle_{+} \geq\left\langle v^{*}, v\right\rangle$ for each $v^{*} \in J(x-w)$, by a well-known characterization of the subdifferential $J$ of $k$ in terms of the directional derivatives of $k$, it is clear that (c) implies (f) (hence that (e) implies (f)). Condition (f) implies (e) again by applying subdifferential calculus. Indeed, the subdifferential at 0 of the sublinear 
functional $s:=p+q$ defined by $p(\cdot):=\langle x-w, \cdot\rangle_{+}, q(\cdot):=i_{T}(\cdot)$, with $T:=T(C, x)$ is given by

$$
\partial s(0)=\partial p(0)+\partial q(0)=J(x-w)+T^{o},
$$

as easily seen, $p$ being continuous (observe that $x^{*} \in \partial q(0)$ iff $x^{*} \mid T \leq 0$ ). As $C-x$ is contained in $T(C, x)$, it is obvious that (f) implies (g). Since $p$ is sublinear continuous, condition (g) implies (f) by density of $T^{r}(C, x)$ into $T(C, x)$. Moreover, Lemma 2.3 yields the equivalence $(\mathrm{g}) \Leftrightarrow(\mathrm{h})$. Finally, as $\langle-x,-y\rangle_{+}=\langle x, y\rangle_{+},(\mathrm{g}) \Leftrightarrow(\mathrm{i})$ and $(\mathrm{h}) \Leftrightarrow(j)$

Example. Let $X=L^{p}(\Omega, \mathbb{R})$, with $1<p<+\infty$, where $(\Omega, \mathcal{A}, \mu)$ is a measured space. For $C \in \mathcal{C}(X)$ and $w \in X$, the vector $x \in C$ is the metric projection of $w$ in $C$ if, and only if,

$$
\int_{\Omega}|x(s)-w(s)|^{p-1} \operatorname{sgn}(x(s)-w(s))(y(s)-x(s)) d \mu(s) \geq 0, \forall y \in C .
$$

This formula is the same as the one proved in [16] by using specific properties of $L^{p}$ spaces.

Let also remark that, except if $k$ is Gâteaux-differentiable, the characterization (g) of Theorem 3.1 cannot be equivalently rewritten as

$$
\langle w-x, y-x\rangle_{+} \leq 0, \forall y \in C, \text { or }\langle x-w, x-y\rangle_{+} \leq 0, \forall y \in C,
$$

as the following counterexample shows:

Example. Let $X=l^{1}(\mathbb{R})$ with $\|x\|=\sum_{i}\left|x_{i}\right|$. One has (see [11])

$$
\langle x, y\rangle_{+}=\left(\|x\|-\sum_{i}\left(\left|x_{i}\right|-x_{i} \operatorname{sgn} y_{i}\right)\right)\|y\| .
$$

It is easily seen that the relation $\langle x, y\rangle_{+} \geq 0$ is not equivalent to the relation $\langle-x, y\rangle_{+} \leq 0$ or $\langle x,-y\rangle_{+} \leq 0$. Indeed, for $x \neq 0$ and $y \neq 0$, one has $\langle x, y\rangle_{+} \neq-\langle-x, y\rangle_{+}$, and $\langle x, y\rangle_{+} \neq-\langle x,-y\rangle_{+}$. In fact, $\langle x,-y\rangle_{+}=$ $\langle-x, y\rangle_{+}=-\langle x, y\rangle_{-}$, where

$$
\langle x, y\rangle_{-}:=-\langle x,-y\rangle_{+}=\min \left\{\left\langle x^{*}, y\right\rangle: x^{*} \in J(x)\right\} .
$$

When $C$ is a closed convex cone, the preceding characterization takes a striking form.

Corollary 3.2. If $C$ is a closed convex cone, $x \in C$ is a projection of $w$ in $C$ if, and only if, there exists $y^{*} \in J(w-x)$ such that $y^{*} \in C^{\circ}$ and $\left\langle y^{*}, x\right\rangle=0$. If $C$ is a closed vector subspace, the vector $x$ is a projection of $w$ in $C$ if, and only if, there exists $y^{*} \in J(w-x)$ such that $\left\langle y^{*}, y\right\rangle=0$ for all $y \in C$.

Proof. This follows immediately from Theorem 3.1 (c) since $u^{*} \in N(C, x)$ if, and only if, $u^{*} \in C^{\circ}$ and $\left\langle u^{*}, x\right\rangle=0$.

The following simple consequence deserves some attention since this property is not necessarily valid when $C$ is not a convex subset. 
Corollary 3.3. Let $C$ be a nonempty closed convex subset of a Banach space $X$. Let $w \in X \backslash C$ and $x \in P(C, w)$. Then, for any $r>0, x$ is a best approximation of $z=x+r(w-x)$.

Proof. This follows from the equivalence $(\mathrm{a}) \Leftrightarrow(\mathrm{c})$ of Theorem 3.1 and the positive homogeneity of $J \cdot \square$

A geometrical interpretation of our characterization of metric projections can be given thanks to the following definition.

Definition 3.4. ([7, p. 71], [24]) The vector $x$ is said to form an obtuse angle with $v$ if one of the following equivalent conditions holds:

(i) $\langle x,-v\rangle_{+} \geq 0$.

(ii) There exists $x^{*} \in J(x)$ such that $\left\langle x^{*}, v\right\rangle \leq 0$.

(iii) The vector $x$ is orthogonal to $-v$ in the sense of Birkhoff:

$$
\|x-t v\| \geq\|x\|, \forall t>0 .
$$

(iv) There exists $r>0$ such that for all $t \in[0, r],\|x-t v\| \geq\|x\|$.

(v) The vector $x$ is normal to $Y:=\mathbb{R}_{-} v$ at 0 in the sense of Bony or metrically normal:

$$
\exists r>0: d_{Y}(0+t(-v))=t\|(-v)\|, \forall t \in[0, r] .
$$

(vi) The vector $x$ is metrically normal to $Z:=[-1,0] v$ at 0 .

By rewriting the equivalence $(\mathrm{a}) \Leftrightarrow(\mathrm{g})$ in Theorem 3.1, one has:

Corollary 3.5. The vector $x \in C$ is a projection of $w$ in $C$ if, and only if, for all $v \in C$, the vector $x-w$ forms an obtuse angle with $x-v$.

In the particular case of Euclidean and Hilbertian spaces, our extension obviously coincides with the usual analytic and geometric Kolmogorov characterization. The semi-inner product and the duality mapping are also welladapted tools to the characterization of metric projections in Banach spaces.

Let us point out that Corollary 3.2 can be reformulated as a decomposition theorem of Moreau type in a Banach space (see [20], [21]). It keeps the main features of the Moreau decomposition in Hilbert spaces, but here the decomposition is not symmetric.

Corollary 3.6. Let $C$ be a proximinal closed convex cone of $X$ (that is, such that each $x \in X$ has a best approximation in $C$ ), and let

$$
D:=J^{-1}\left(C^{\circ}\right)=\left\{y \in X: J(y) \cap C^{\circ} \neq \emptyset\right\} .
$$

Then, any $w \in X$ can be decomposed as

$$
w=x+y, x \in C, y \in D, y \perp x,
$$

where $y \perp x$ means there exists $y^{*} \in J(y)$ with $\left\langle y^{*}, x\right\rangle=0$; in fact, one can also impose $x \in P(C, w)$.

Note that $D$ is a closed cone but its convexity is not ensured in general. 
Example. Let $C=\mathbb{R}_{+}(-1,-1)$ be the closed convex cone generated by $-e=(-1,-1)$ in the Banach space $X=\mathbb{R}^{2}$, whose norm is chosen to be the $l^{1}$ norm: for $z=(x, y),\|z\|=|x|+|y|$. Then one has

$$
J(z)=\|z\|(\partial|.|(x) \times \partial|.|(y)) \text { and } C^{\circ}=\left\{(u, v) \in \mathbb{R}^{2}: v \geq-u\right\} .
$$

A simple calculus gives that $J^{-1}\left(C^{\circ}\right)=\left(\mathbb{R}_{+} \times \mathbb{R}\right) \cup\left(\mathbb{R} \times \mathbb{R}_{+}\right)$which is not convex.

We will see that in important cases $D:=J^{-1}\left(C^{\circ}\right)$ is a closed convex cone, in particular that $D$ satisfies the equality $D=-C$. Let us observe that the two inclusions we need have been characterized in [32]. We provide a direct proof of the first one for completeness and for later use.

Lemma 3.7. [32] The norm of a Banach space $X$ is nondecreasing on a closed convex cone $C$, in the sense that $\|x\| \leq\|y\|$ whenever $x \in C, y \in x+C$, if, and only if, $-C \subset D:=J^{-1}\left(C^{\circ}\right)$.

Proof. Suppose the function $k$ is nondecreasing on $C$. Then, for any $x, v$ in $C$, we get

$$
k^{\prime}(x, v)=\lim _{t \rightarrow 0_{+}} \frac{1}{t}(k(x+t v)-k(x)) \geq 0 .
$$

Thus, one has

$$
i_{C}(.)+k^{\prime}(x, .) \geq 0=i_{C}(0)+k^{\prime}(x, 0) .
$$

The sum rule for subdifferentials ensures there exists $x^{*} \in \partial i_{C}(0)=C^{\circ}$ such that $-x^{*} \in J(x)=\partial k(x)$. Thus $-x \in J^{-1}\left(x^{*}\right) \subset D$. Conversely, suppose that $-C \subset D$. Whenever $x, v$ are in $C$, by taking $x^{*} \in J(x) \cap\left(-C^{\circ}\right)$, one sees that $k^{\prime}(x, v) \geq\left\langle x^{*}, v\right\rangle \geq 0$. Thus, by the Mean Value Theorem, the function $k$ is nondecreasing on $C$.

As $k^{\prime}(x, y)=\langle x, y\rangle_{+}$, Lemma 3.7 can be reformulated as follows.

Lemma 3.8. The norm of a Banach space $X$ is nondecreasing on a closed convex cone $C$ if, and only if, for all $x, y$ in $C,\langle x, y\rangle_{+} \geq 0$.

Notice that if the norm of $X$ is nondecreasing on the closed convex cone $C$, one also has $\langle x,-y\rangle_{+} \leq 0$, for all $(x, y) \in i n t C \times C$ as easily seen in [25].

The reverse inclusion $J^{-1}\left(C^{\circ}\right) \subset-C$ is also interesting since it shows that whenever $C$ is proximinal, any $w \in X$ can be written as $w=x-y$ with $x \in C, y \in C, y \perp x$. Let us give some conditions to ensure this inclusion.

Proposition 3.9. [32, 1.3] Let $C$ be a closed convex cone of a Banach space $X$. Then,

$J^{-1}\left(C^{\circ}\right) \subset-C \Leftrightarrow\left\{x \in X: d_{C}(-x)=\|x\|\right\} \subset C \Leftrightarrow \forall x \in C, 0 \in P(-C, x)$.

Example. Let $X=L^{1}(\Omega, \mathbb{R})$, where $(\Omega, \mathcal{A}, \mu)$ is a measured space. Let $C:=L^{1}\left(\Omega, \mathbb{R}_{+}\right)$be the cone of nonnegative integrable functions on $\Omega$. If $x$ satisfies $d_{C}(-x)=\|x\|$ or

$$
\int_{\Omega}|x(s)| d \mu(s) \leq \int_{\Omega}|x(s)+y(s)| d \mu(s),
$$


for all $y \in C$, then by taking $y=x^{-}$, one has $x(s) \geq 0$ a.e. $s \in \Omega$, hence $x \in C$. Thus Proposition 3.9 applies. An alternative proof of the inclusion $J^{-1}\left(C^{\circ}\right) \subset-C$ consists in using the characterization of the duality mapping $J$ given in a preceding example which ensures that

$$
x \in J^{-1}\left(C^{\circ}\right) \Leftrightarrow \mu([x>0])=0 \Leftrightarrow x \in-C .
$$

Proposition 3.10. $[32,1.5]$ Let $C$ be a closed convex cone of a reflexive and strictly convex Banach space $X$. The dual norm of $X$ is nondecreasing on $C^{*}:=-\left(C^{\circ}\right)$ if, and only if $J^{-1}\left(C^{\circ}\right) \subset-C$.

Example. Let $X=L^{p}(\Omega, \mathbb{R})$, where $1<p<+\infty$ and $(\Omega, \mathcal{A}, \mu)$ is a measured space. Let $C:=L^{p}\left(\Omega, \mathbb{R}_{+}\right)$be the cone of nonnegative $p$-integrable functions on $\Omega$. The norm of $X$ is nondecreasing on $L^{p}\left(\Omega, \mathbb{R}_{+}\right)$, and the norm of $X^{*}$ is nondecreasing on $L^{q}\left(\Omega, \mathbb{R}_{+}\right)$, with $\frac{1}{p}+\frac{1}{q}=1$, thus Proposition 3.10 applies. An alternative proof of the inclusion $J^{-1}\left(C^{\circ}\right) \subset-C$ consists in using the characterization of the duality mapping $J$ given in a preceding example which ensures that

$$
x \in J^{-1}\left(C^{\circ}\right) \Leftrightarrow \operatorname{sgn} x \leq 0 \Leftrightarrow \mu([x>0])=0 \Leftrightarrow x \in-C .
$$

The following result is a partial extension of Proposition 3.10 as reflexivity is not supposed here.

Proposition 3.11. Let $C$ be a closed convex cone of a Banach space $X$. Suppose the dual norm of $X$ is smooth off 0 and nondecreasing on $C^{*}:=$ $-\left(C^{\circ}\right)$. Then $J^{-1}\left(C^{\circ}\right) \subset-C$.

Proof. Let $x \in D:=J^{-1}\left(C^{\circ}\right)$ : there exists $x^{*} \in J(x) \cap C^{\circ}$. Then $x \in$ $J^{*}\left(x^{*}\right)$, where $J^{*}$ is the duality mapping of $X^{*}$. Since the dual norm of $X^{*}$ is smooth off $0, J^{*}\left(x^{*}\right)$ is a singleton. Moreover, since the dual norm is nondecreasing on $C^{*}$, we have by Lemma 3.7

$$
C^{\circ}=-C^{*} \subset\left(J^{*}\right)^{-1}\left(\left(C^{*}\right)^{\circ}\right)=-\left(J^{*}\right)^{-1}\left(C^{\circ \circ}\right),
$$

hence $x=J^{*}\left(x^{*}\right) \in\left(C^{*}\right)^{\circ}=-C^{\circ \circ}$ (bipolar in $X^{* *}$ ), thus $-x \in C^{\circ \circ} \cap X=$ C.

Gathering Corollary 3.2, Lemma 3.7 and Proposition 3.11, we obtain the following decomposition.

Corollary 3.12. Suppose $X$ is a normed vector space whose dual norm is smooth off 0 and suppose $C$ is a proximinal closed convex cone in $X$ such that the associated preorder is a lattice order. Assume the norm of $X$ is nondecreasing on $C$ and the dual norm is nondecreasing on $C^{*}:=-\left(C^{\circ}\right)$. Then for $D:=J^{-1}\left(C^{\circ}\right)$, any $w$ can be decomposed as $w=x+y$ with $x \in C$, $y \in D=-C, y \perp x$.

Another decomposition can be obtained when the space is a dual space.

Corollary 3.13. Suppose $X$ is the dual space of a Banach space $X_{*}$ ordered by a closed convex cone $C_{*}$. Let $C=-C_{*}^{\circ}$. If the norm of $X_{*}$ is nondecreasing on $C_{*}$, then each $w \in X$ can be decomposed as $w=x+y$ with $x \in P(C, w), y \in-C \cap J^{-1}\left(C^{\circ}\right), y \perp x$. 
Proof. It is shown in [26, Th. 3.8], that when the norm of $X_{*}$ is nondecreasing on $C_{*}$, then each $f \in X$ can be written as $f=f_{+}-f_{-}$with $f_{+}, f_{-} \in C$ and $\left\|f_{+}\right\|=d_{C}(-f)$. Taking $f=-w$, setting $x=f_{-}, y=-f_{+}$and observing that

$$
\|w-x\|=\|y\|=\left\|f_{+}\right\|=d_{C}(w),
$$

we get that $x \in P(C, w)$ and $y \in-C$. The relations $y \in J^{-1}\left(C^{\circ}\right), y \perp x$ are provided by Corollary 3.6.

It will be shown in the next section that, when $X$ is a vector lattice for the preorder defined by a closed convex cone $C$ of $X$, this decomposition can be made more precise in the sense that it corresponds to an order decomposition $w=w^{+}-w^{-}$, with $w^{+} \in P(C, w),-w^{-} \in P(-C, w)$, and $w^{+} \perp w^{-}$.

\section{An application to ordered Banach spaces}

Let $C$ be a nonempty closed convex cone in $X$, and let $\leq$ be the preorder defined by the cone $C$ in the usual sense: for all $x, y \in X, x \leq y$ if and only if $y-x \in C$. We assume that $X$ is a vector lattice with respect to this preorder and we set: $x^{+}:=\sup (x, 0), x^{-}:=\sup (-x, 0),|x|=\sup (x,-x)$. In this case, (see [28]), one has $x=x^{+}-x^{-}$.

Corollary 4.1. Let $w \in X$. If the following conditions hold:

(i) For all $u \in C,\left\langle w^{-}, u\right\rangle_{+} \geq 0$,

(ii) $\left\langle w^{-}, w^{+}\right\rangle_{+} \leq 0$,

then the vector $w^{+}$is a metric projection of $w$ in $C$, that is $\left\|w^{-}\right\|=d_{C}(w)$. Moreover, if the function $k$ is Gâteaux-differentiable at $w^{-}$, the converse holds.

Proof. This result is a consequence of Theorem 3.1 (f) since (i) and (ii) imply $\left\langle w^{-}, w^{+}\right\rangle_{+} \leq 0$ and since $\left\langle w^{-}, .\right\rangle_{+}$is sublinear.

Since for all $v, w \in X$, the implication $(v \leq w) \Rightarrow\left(v^{+} \leq w^{+}\right)$holds by definition of a supremum, the metric projection thus obtained is homotone (or order preserving, or isotone, see [14]). Moreover, we recall that condition (i) holds whenever the norm is nondecreasing on $C$ (thanks to Lemma 3.8) and in particular $\left\langle w^{-}, w^{+}\right\rangle_{+} \geq 0$. We may also remark that whenever assumption (i) holds, assumption (ii) can be replaced by $\left\langle w^{-}, w^{+}\right\rangle_{+}=0$.

The following terminology will be convenient.

Definition 4.2. The closed convex cone $C$ is said to be "obtuse" if

$$
\forall(x, y) \in C \times C, \inf (x, y)=0 \Rightarrow\langle x, y\rangle_{+} \leq 0 .
$$

The closed convex cone $C$ is said to be "orthogonal" if

$$
\forall(x, y) \in C \times C, \inf (x, y)=0 \Rightarrow\langle x, y\rangle_{+}=0 .
$$

Lemma 3.8 implies that both notions coincide when the norm is nondecreasing on $C$. 
Example. The set $C=\left\{(r \cos \theta, r \sin \theta): r \in \mathbb{R}_{+}, \theta \in\left[0, \frac{3 \pi}{4}\right]\right\}$ is obtuse but non orthogonal in the Euclidean space $X=\mathbb{R}^{2}$. Indeed the Euclidean norm is not nondecreasing on $C$ since $\|(0,1)\|_{2}<\|(-1,1)\|_{2}$ although $(0,1)-$ $(-1,1) \in C$.

The following proposition gives an useful property of obtuse cones.

Proposition 4.3. The following assertions are equivalent.

(i) The closed convex cone $C$ is obtuse.

(ii) $\forall w \in X,\left\langle w^{-}, w^{+}\right\rangle_{+} \leq 0$.

(iii) $\forall w \in X,\left\langle w^{+}, w^{-}\right\rangle_{+} \leq 0$.

Proof. It suffices to prove that (i) $\Leftrightarrow$ (ii), the equivalence (i) $\Leftrightarrow$ (iii) being obtained by changing $w$ into $-w$. Assertion (i) implies (ii). Indeed, for all $w \in X$, one has $\inf \left(w^{-}, w^{+}\right)=0$. Conversely, suppose (ii) holds. Let $(x, y) \in C \times C$ with $\inf (x, y)=0$. Setting $w=y-x$, one has (see [28])

$$
w^{-}=-\inf (y-x, 0)=-(\inf (x, y)-x)=x \text { and } w^{+}=w+w^{-}=y .
$$

Using assertion (ii), one has

$$
\langle x, y\rangle_{+}=\left\langle w^{-}, w^{+}\right\rangle_{+} \leq 0 .
$$

Thus the cone $C$ is obtuse.

A similar proof, in which the relation $\leq$ is replaced by $=$, yields the following statement.

Proposition 4.4. The following assertions are equivalent:

(i) The closed convex cone $C$ is orthogonal.

(ii) $\forall w \in X,\left\langle w^{-}, w^{+}\right\rangle_{+}=0$.

(iii) $\forall w \in X,\left\langle w^{+}, w^{-}\right\rangle_{+}=0$.

Now, let us give the first main result of this section. It is an extension to general Banach spaces, not necessarily Hilbertian, of a result stated by Isac [14, Th 4.2] about the behaviour of metric projections.

Theorem 4.5. Suppose a Banach space $X$ is a vector lattice with respect to the preorder $\leq$ defined by a closed convex cone $C$ of $X$. Assume the norm of $X$ is nondecreasing on $C$. If $C$ is obtuse then for each $w \in X, w^{+}$is a metric projection of $w$ in $C$, that is $\left\|w^{-}\right\|=d_{C}(w)$. Moreover, if the function $k$ is Gâteaux-differentiable on $X$, the converse holds.

Proof. Let $w \in X$. Assume the norm of $X$ is nondecreasing on $C$. First of all, if we suppose that the cone $C$ is obtuse (in this case it is orthogonal), we deduce from Corollary 4.1 and Lemma 3.8 that $w^{+}$is a metric projection of $w$ in $C$, that is $\left\|w^{-}\right\|=d_{C}(w)$. Conversely, assume the function $k$ is Gâteaux-differentiable at $w^{-}$and $w^{+}$is a metric projection of $w$ in the cone $C$. Since $0 \in C$, one has by Theorem $3.1(\mathrm{~g})$ and the linearity of $\left\langle w^{-}, .\right\rangle_{+}$,

$$
0 \geq-\left\langle w^{+}-w, 0-w^{+}\right\rangle_{+}=-\left\langle w^{-},-w^{+}\right\rangle_{+}=\left\langle w^{-}, w^{+}\right\rangle_{+} \geq 0 .
$$

Thus, by Proposition 4.4, the cone $C$ is orthogonal. 
Example. If $X=L^{1}(\Omega, \mathbb{R})$, where $(\Omega, \mathcal{A}, \mu)$ is a measured space, then for each $w \in X, w^{+}$is a projection of $w$ in the positive cone $C=L^{1}\left(\Omega, \mathbb{R}_{+}\right)$ as easily seen in [25] although $k$ is not Gâteaux-differentiable and $C$ is not orthogonal. In fact, $\left\langle w^{-}, w^{+}\right\rangle_{+}=\left\|w^{-}\right\|\left\|w^{+}\right\|$as easily seen either from the expression of the semi-inner product given above, else from $\left\langle w^{-}, w^{+}\right\rangle_{+}=$ $\left.\frac{d}{d t}\right|_{t=0}\left\|w^{-}+t w^{+}\right\|$.

In the sequel, we will make use of the following definition.

Definition 4.6. [28] The norm of the vector lattice $X$, with respect to the preorder $\leq$ defined by a closed convex cone $C$ of $X$, is said to be absolute if $\||x|\|=\|x\|$ for all $x$.

Proposition 4.7. Suppose a Banach space $X$ is a vector lattice with respect to the preorder $\leq$ defined by a closed convex cone $C$ of $X$. Assume the norm of $X$ is Gâteaux-differentiable off 0 and absolute. Then, the closed convex cone $C$ is orthogonal.

Proof. It is known (see [28]) that

$$
|x-y|=\sup (x, y)-\inf (x, y) \text { and } x+y=\sup (x, y)+\inf (x, y) .
$$

Hence, if $x$ and $y$ belong to $C$ with $\inf (x, y)=0$, then $|x-y|=x+y$. For $t \in] 0,1], 0 \leq t y \leq y$, so inf $(x, t y)=\inf (x, y)=0$, thus $|x-t y|=x+t y$. Since the norm is absolute, one has

$$
\|x-t y\|=\||x-t y|\|=\|x+t y\| .
$$

It follows from the definition of the semi-inner product that $\langle x,-y\rangle_{+}=$ $\langle x, y\rangle_{+}$. As the norm is Gâteaux-differentiable off 0 , we obtain $2\langle x, y\rangle_{+}=0$. By changing the role of $x$ and $y,\langle y, x\rangle_{+}=0$ as well, thus the cone $C$ is orthogonal.

Our assumptions are in particular satisfied when one studies the classical framework of Banach lattices in the usual sense.

Definition 4.8. The space $X$ is called a Banach lattice, with respect to an order $\leq$, if $X$ is a Banach space satisfying the following assumptions:

(i) $X$ is a vector lattice with respect to $\leq$.

(ii) $0 \leq x \leq y$ implies that $\|x\| \leq\|y\|$ for all $x, y$.

(iii) The norm of $X$ is absolute.

Theorem 4.9. Assume $X$ is a Banach lattice, with respect to the preorder $\leq$ defined by a closed convex cone $C$ of $X$. If the function $k$ is Gâteauxdifferentiable on $X$, then for all $w \in X, w^{+}$is a metric projection of $w$ in C.

Proof. It suffices to apply Theorem 4.5, Definition 4.8, and Proposition 4.7.

Let us recall that a Hilbert lattice is a Banach lattice with an Hilbertian norm. It is easily seen that the following result, established by Isac in Hilbert spaces (see [14, Th. 4.2]), is an immediate consequence of the preceding theorem. 
Corollary 4.10. [14, Th. 4.2] If the space $X$ is a Hilbert lattice with respect to the order $\leq$ defined by a closed convex cone $C$ of $X$, then for all $w \in X$, $w^{+}$is the metric projection $p_{C}(w)$ of $w$ in $C$.

Let us illustrate these results by some examples.

Example. Let $X=L^{p}(\Omega, \mathbb{R})$, with $1<p<+\infty$, where $(\Omega, \mathcal{A}, \mu)$ is a measured space. Let $C:=L^{p}\left(\Omega, \mathbb{R}_{+}\right)$be the cone of nonnegative $p$-integrable functions on $\Omega$. Obviously, for $w \in X, w^{+}$is given by

$$
w^{+}=\sup (w, 0) \Leftrightarrow w^{+}(s)=\sup (w(s), 0)=w(s)^{+} \text {a.e. } s \in \Omega .
$$

Then, $w^{+}$is the metric projection of $w$ in $C$. Indeed, $L^{p}(\Omega, \mathbb{R})$ is a Banach lattice with a Gâteaux-differentiable norm off 0 , and Theorem 4.9 applies. This result can also be proved by using the specific properties of $L^{p}$-spaces (see [25]). Notice that it can be shown directly that the cone $C$ is orthogonal by using the semi-inner product:

$$
\left\langle w^{-}, w^{+}\right\rangle_{+}=\left\|w^{-}\right\|^{2-p} \int_{\Omega}\left(w^{-}(s)\right)^{p-1} w^{+}(s) d \mu(s)=0 .
$$

The following examples, for which the norm is not necessarily differentiable, satisfy the assumptions of Theorem 4.5.

Example. Given a topological compact space $T$, let us consider, as before, $X=C(T)$. Let $C:=C\left(T, \mathbb{R}_{+}\right)$be the cone of nonnegative continuous functions on $T$. The space $X$ is known to be a Banach lattice with respect to the order $\leq$ defined by $C$ (see [28]), and one has

$$
x^{+}=\sup (x, 0) \Leftrightarrow x^{+}(t)=\sup (x(t), 0)=x(t)^{+} \forall t \in T,
$$

We claim that $w^{+}$is a metric projection of $w$ in $C$. In fact, by applying Theorem 4.5, it suffices to verify that the cone $C$ is orthogonal:

$$
\left\langle w^{+}, w^{-}\right\rangle_{+}=\max \left\{w^{-}(t) w^{+}(t): t \in T\left(w^{+}\right)\right\}=0,
$$

since

$$
T\left(w^{+}\right):=\left\{t \in T:\left|w^{+}(t)\right|=\|w\|\right\} \subset\left\{t \in T: w^{-}(t)=0\right\}
$$

if $w^{+} \neq 0$, and $\left\langle w^{-}, w^{+}\right\rangle_{+}=0$, if $w^{+}=0$. This result can also be proved by using the specific properties of $C(T)$-spaces (see [25]).

Example. Let $X:=\mathbb{R}^{d}$ equipped with the norm $\|x\|_{\infty}:=\max _{1 \leq i \leq d}\left|x_{i}\right|$, and let $C:=\mathbb{R}_{+}^{d}$ be the positive cone of $\mathbb{R}^{d}$. In this case

$$
\begin{gathered}
0 \leq x \leq y \Rightarrow \max _{1 \leq i \leq d} x_{i}=\|x\|_{\infty} \leq\|y\|_{\infty}=\max _{1 \leq i \leq d} y_{i}, \\
\left\langle w^{-}, w^{+}\right\rangle_{+}=\left\|w^{-}\right\| \max _{i \in I\left(w^{-}\right)} w_{i}^{+}=0,
\end{gathered}
$$

where $I\left(w^{-}\right)=\left\{1 \leq i \leq d: w_{i}^{-}=\left\|w^{-}\right\|_{\infty}\right\}$. Thus, applying Theorem 4.5, $w^{+}=\left(w_{i}^{+}\right)_{1 \leq i \leq n}$ is a metric projection of $w$ in $C$ for the supremum norm. 
Example. Let $X:=L_{s}(E)$ be the space of symmetric linear operators in a finite dimensional Euclidean space $E$ with $\operatorname{dim} E=d$, and with inner product (.|.). Let $C:=L_{s}^{+}(E)$ be the cone of semi-definite positive elements of $L_{s}(E)$. For any matrix $A \in X$, it is known there exists an orthogonal matrix $U$ and a diagonal matrix $D=\operatorname{diag}\left(\lambda_{1}, \ldots, \lambda_{d}\right)$, where $\lambda_{i}$ are the eigenvalues of $A$, such that $A=U^{-1} D U$. On the space $L_{s}(E)$, let us consider a norm satisfying the following monotonicity property:

$$
0 \leq A \leq B \Rightarrow\|A\| \leq\|B\|,
$$

In this case, the space $X$ is a vector lattice with respect to the preorder $\leq$ (see [18]), and one has for $A \in X$

$$
\begin{gathered}
|A|=\left(A^{2}\right)^{\frac{1}{2}}=U^{-1}\left(D^{2}\right)^{\frac{1}{2}} U ; \\
A^{+}:=\sup (A, 0)=\frac{1}{2}(|A|+A) ; A^{-}:=\sup (-A, 0)=\frac{1}{2}(|A|-A) ; \\
A^{+}=U^{-1} D^{+} U, A^{-}=U^{-1} D^{-} U,
\end{gathered}
$$

where $D^{+}=\operatorname{diag}\left(\lambda_{1}^{+}, \ldots, \lambda_{d}^{+}\right)$and $D^{-}=\operatorname{diag}\left(\lambda_{1}^{-}, \ldots, \lambda_{d}^{-}\right)$. In particular, when $\|\cdot\|$ is the Frobenius norm given by

$$
\|A\|:=\sqrt{\sum_{i, j=1, \ldots, d} a_{i, j}^{2}}=\sqrt{\operatorname{tr} A^{2}},
$$

the space $X$ equipped with this absolute norm is Euclidean with inner product given by $((A \mid B))=\operatorname{tr}(A B)$. It is also a Hilbert lattice. Here, we can check directly that the cone $C$ is orthogonal:

$$
\left\langle A^{-}, A^{+}\right\rangle_{+}=\left(\left(A^{-} \mid A^{+}\right)\right)=\operatorname{tr}\left(A^{-} A^{+}\right)=\operatorname{tr}\left(U^{-1} D^{-} D^{+} U\right)=0 .
$$

Thus, Corollary 4.10 ensures that $A^{+}$is the metric projection of $A$ in $C$ for the Frobenius norm. Unfortunately, Theorem 4.9 or Corollary 4.10 cannot be applied if we consider the supremum norm given by

$$
\|A\|:=\max _{v \in S_{X}}|(A v \mid v)|=\max \left(\left|\lambda_{1}\right|, \ldots,\left|\lambda_{d}\right|\right)=\|\lambda\|_{\infty} \text { where } \lambda=\left(\lambda_{1}, \ldots, \lambda_{d}\right) .
$$

However, Theorem 4.5 enables us to conclude. Indeed, the norm is nondecreasing on $C$ and the cone $C$ is orthogonal since

$$
\left\langle A^{-}, A^{+}\right\rangle_{+}=\left\|A^{-}\right\| \max _{i \in I\left(\lambda^{-}\right)} \lambda_{i}^{+}=0
$$

where

$$
I\left(\lambda^{-}\right)=\left\{1 \leq i \leq d: \lambda_{i}^{-}=\left\|\lambda^{-}\right\|_{\infty}\right\} .
$$

Thus, Theorem 4.5 ensures that $A^{+}$is a metric projection of $A$ in $C$ for the supremum norm.

A partial extension of what precedes to the infinite dimensional case is possible. If $E$ is a separable Hilbert space, and $X$ is the space of HilbertSchmidt operators $A$ on $E$, then there exists a sequence $\left(\lambda_{n}\right)_{n \in \mathbb{N}}$ of reals with $\left|\lambda_{0}\right| \leq\left|\lambda_{1}\right| \ldots \leq\left|\lambda_{n}\right| \leq \ldots$ and an Hilbertian basis $\left(u_{n}\right)_{n \in \mathbb{N}}$ of $E$, such 
that for all $A \in X$, one has $A u_{n}=\lambda_{n} u_{n}$. So by using the Hilbert-Schmidt norm given by

$$
\|A\|:=\left(\sum_{n \in \mathbb{N}}\left(A u_{n} \mid A u_{n}\right)\right)^{\frac{1}{2}}=\left(\sum_{n \in \mathbb{N}}\left|\lambda_{n}\right|^{2}\right)^{\frac{1}{2}},
$$

we get that the cone $C$ is orthogonal since

$$
\left\langle A^{-}, A^{+}\right\rangle_{+}=\sum_{n \in \mathbb{N}}\left(A^{-} u_{n} \mid A^{+} u_{n}\right)=\sum_{n \in \mathbb{N}}\left(\lambda_{n}^{-} u_{n} \mid \lambda_{n}^{+} u_{n}\right)=0 .
$$

Since the Hilbert-Schmidt norm is nondecreasing on $C$, Corollary 4.10 holds, and $A^{+}$is then the metric projection of $A$ in $C$ for the Hilbert-Schmidt norm.

\section{AN APPLICATION TO VARIATIONAL INEQUALITIES}

In this section we apply the characterizations of metric projections given in Theorem 3.1 (c) to variational inequalities. Let us first recall that if $X$ and $Y$ are two topological vector spaces, a multimapping $M$ from $X$ into $Y$ is said to be surjective if

$$
\forall y \in Y, \exists x \in X: y \in M(x) .
$$

Moreover, the inverse image of a multimapping $M$ is given for a subset $Z \subset Y$ by

$$
M^{-1}(Z)=\{x \in X: Z \cap M(x) \neq \emptyset\} .
$$

Now, let us take a Banach space $X$, an application $A$ from $X$ into $X^{*}$, a linear continuous form $f \in X^{*}$ and $C$ a nonempty closed convex subset of $X$. Let us consider the variational inequality

Find $x \in C$ such that

$$
\langle A x-f, y-x\rangle \geq 0, \forall y \in C .
$$

This variational inequality can be rewritten as a generalized equation:

$$
\text { Find } x \in C \text { such that } f-A x \in N(C, x) \text {. }
$$

The following observation is just a consequence of the definition of the duality mapping $J$. If $x \in C$ is a solution of the variational inequality $(V I)$, and if the element $A x-f$ of $X^{*}$ attains its norm, then for each $u_{x} \in J^{-1}(A x-f)$ one has

$$
f-A x \in J\left(u_{x}\right) \cap N(C, x) .
$$

As observed in [22], the variational inequality $(V I)$ may also be seen in terms of metric projections. The following proposition generalizes the result given in [22] in which it is supposed that the space $X$ is smooth (hence that $J$ is single-valued), and that the space is rotund (hence that the metric projection is unique). Its corollary yields a characterization of solutions of the variational inequality $(V I)$. It has been kindly pointed to us by Professor Y. Al'ber, when editing the present paper, that a similar statement has been announced in reference [2]. Let us note however that the assumption of Corollary 5.2 below does not impose that $J$ is everywhere single-valued. 
Proposition 5.1. Assume that $f-A x \in J(u)$ for some $x \in C$, and for some $u \in X$. Then, among the followings conditions, one has the implications $(i) \Rightarrow(i i) \Leftrightarrow(i i i) \Leftrightarrow(i v) \Rightarrow(v) \Leftarrow(v i) \Leftarrow(i)$

(i) $f-A x \in N(C, x)$

(ii) For all $r>0, x \in P(C, x+r u)$;

(iii) For some $r>0, x \in P(C, x+r u)$;

(iv) $0 \in P(T(C, x), u)$;

(v) $\langle A x-f, v\rangle \leq 0, \forall v \in P(T(C, x), u)$;

(vi) $\langle A x-f, v\rangle=0, \forall v \in P(T(C, x), u)$;

Proof. 1) Let us prove (i) $\Rightarrow$ (ii). Since by hypothesis, $f-A x \in N(C, x)$, and $r(f-A x) \in J(r u)$ for all $r>0$, we get by Theorem 3.1 (e) that $x \in P(C, x+r u)$.

2) The equivalence between (ii) and (iii) is a direct consequence of Corollary 3.3

3) (iii) is equivalent to (iv). Namely $0 \in P(T(C, x), u)$ if and only if

$$
\exists z^{*} \in J(0-u):\left\langle z^{*}, v-0\right\rangle \geq 0 \forall v \in T(C, x) .
$$

In turn, by the equivalence $(\mathrm{a}) \Leftrightarrow(\mathrm{c})$ of Theorem 3.1 , this assertion means that $x \in P(C, x+u)$; taking into account the equivalence of (ii) with (iii) we get that (iii) is equivalent to (iv).

4) Now, let us prove that (iv) implies (v). Let $v \in P(T(C, x), u)$. As $0 \in T(C, x)$, one has

$$
\begin{gathered}
\|u\|=\|u-0\| \geq\|u-v\| \\
\langle A x-f, v-u\rangle+\langle A x-f, u\rangle \leq\|A x-f\|\|u-v\|-\|u\|^{2}, \\
\langle A x-f, v\rangle \leq\|u\|\|u-v\|-\|u\|^{2} \leq 0 .
\end{gathered}
$$

5) Let us assume (i) holds, so that for each $v \in T(C, x)$ we already have $\langle A x-f, v\rangle \geq 0$. As (i) implies (v) we also have the reverse inequality for each $v \in P(T(C, x), u)$, so that (vi) holds.

Corollary 5.2. Given $x \in C$ such that for some $u \in J^{-1}(f-A x), J(u)$ is a singleton, the following assertions are equivalent:

(i) $x \in C$ is a solution of the variational inequality $(V I)$;

(ii) For all $r>0, x \in P(C, x+r u)$;

(iii) For some $r>0, x \in P(C, x+r u)$.

Proof. The implications (i) $\Rightarrow$ (ii) $\Rightarrow$ (iii) are consequences of Proposition 5.1 since $f-A x \in N(C, x)$ when $x \in C$ is a solution of the variational inequality $(V I)$. Now, if (iii) holds, Theorem 3.1 (e) yields

$$
J((x+r u)-x) \cap N(C, x) \neq \emptyset .
$$

Since $J(r u)=r J(u)$ is the singleton $f-A x$, we have $f-A x \in N(C, x) \cap J(u)$ and (i) is satisfied by the reformulation of $(V I)$ given above.

In the following corollary we use more concrete assumptions. 
Corollary 5.3. Suppose that for $x$ given in $X$,

(i) $A x-f \in J(X)$.

(ii) For some $u \in J^{-1}(A x-f)$, the function $k$ is Gâteaux-differentiable at $u$.

Then $x \in C$ is a solution of the variational inequality $(V I)$ if, and only if, for some $r>0, x \in P(C, x+r u)$. In particular, if $X$ is the dual space of a strictly convex Banach space $X_{*}$, if $f \in X_{*}$, and if $A$ maps $X$ into $X_{*}$, the preceding equivalence holds.

\section{REFERENCES}

[1] Ya. I. Al'ber and A.I. Notik. Parallelogram inequalities in Banach spaces and some properties of the duality mapping, Ukrainian Math. J. 40 (1988), 650-652.

[2] Ya. I. Al'ber, Generalized projection operators in Banach spaces: properties and applications, in Functional Differential Equations, Proceedings of the Israel Seminar, Vol. I, Ariel, Israel, 1994, pp. 5-10.

[3] Ya. I. Al'ber, Metric and generalized projection operators in Banach spaces: properties and applications, in Theory and Applications of Nonlinear Operators of Monotone and Accretive Types, (ed. A. G. Kartsatos), Marcel Dekker, E-preprint funct-an/9311001, 1996, pp. $15-50$.

[4] Ya. I. Al'ber, A bound for the modulus of continuity for metric projections in a uniformly convex and uniformly smooth Banach spaces, Journal Approx. Theory, 85 (1996), 199-223.

[5] E. Asplund and R. T. Rockafellar, Gradients of convex functions, Trans. Amer. Math. Soc. 139 (1969), 443-467.

[6] D. Azé, Eléments d'analyse convexe et variationnelle, Ellipse, Editions Marketing SA, Paris, 1997.

[7] V. Barbu, Nonlinear semigroups and differential equations in Banach spaces, Noordhoff, Leyden, 1976.

[8] B. Beauzamy, Introduction to Banach Spaces and their geometry, Mathematic Studies \#68, North-Holland, 1985.

[9] H. Brézis, Analyse fonctionnelle, Masson, Paris, 1985.

[10] R. Dautray and J. L. Lions, Analyse mathématique et calcul numérique pour les sciences et techniques, \#8, Evolution, Masson, Paris, 1984.

[11] K. Deimling, Nonlinear functional analysis, Springer-Verlag, Berlin, 1985.

[12] J. Diestel, Geometry of Banach spaces - selected topics, Lecture Notes in Math. \#485, Springer-Verlag, Berlin, 1975.

[13] J.-B. Hiriart-Urruty and C. Lemaréchal, Convex analysis and minimization algorithms, Springer-Verlag, Berlin, 1993.

[14] G. Isac and B. Nemeth, Isotone Projection Cones in Hilbert Spaces and the Complementarity Problem, Bolletino U.M.I, 7 4.b (1990), 773-802.

[15] V. I. Istratescu, Inner product structures, Reidel, 1987.

[16] D. Landers and L. Rogge, Isotonic Approximation in $L_{s}$, J. Approx. Theory, 31 (1981), 199-223.

[17] G. Lumer, Semi-inner product spaces, Trans. Amer. Math. Soc. 100 (1961), 29-43.

[18] L. A. Lusternik and V. J. Sobolev, Elements of functional analysis, International Monographs on Advanced Mathematics and Physics, Hindustan Publishing Corp., Delhi; Halsted Press [John Wiley \& Sons, Inc.], New York, 1974, 360 pp.

[19] G. J. Minty, On the generalization of a direct method of the calculus of variations, Bull. Amer. Math. Soc. 73 (1967), 315-321.

[20] J. J. Moreau, Décomposition orthogonale d'un espace hilbertien selon deux cônes mutuellement polaires, C. R. Acad. Sci. Paris, Série A Math., 255 (1962), 238-240. 
[21] J. J. Moreau, Proximité et dualité dans un espace hilbertien, Bull. Soc. Math. France, 93 (1965), 273-299.

[22] R. R. Phelps, Metric projections and the gradient projection method in Banach spaces, SIAM J. Control Optim. 23 (1985), 973-977.

[23] R. R. Phelps, Convex functions, monotone operators and differentiability, Lecture Notes in Mathematics, \#1364, Springer-Verlag, Berlin, 1988.

[24] J. P. Penot, A characterization of tangential regularity, Nonlinear Anal. 25 (1981), 625-643.

[25] R. Ratsimahalo, Etude de la projection métrique dans les espaces de Banach, Thesis, Univ. Pau, France, 1996.

[26] D. W. Robinson and S. Yamamuro, The canonical half-norm, dual half-norms, and monotonic norms, Tôhoku Math. J. 35 (1983), 375-386.

[27] R. T. Rockafellar, Convex analysis, Princeton Univ. Press, Princeton, 1970.

[28] H. H. Schaefer, Banach lattices and positive operators, Springer-Verlag, Berlin, 1974.

[29] Y. Sonntag, Convergence au sens de Mosco; théorie et applications à l'approximation des solutions d'inéquations, Thesis, Univ. Provence, Marseille, France, 1982.

[30] R. A. Tapia, A characterization of inner product spaces, Bull. Amer. Math. Soc. 79 (1973), 530-531.

[31] J. J. Vrabie, Compactness methods for nonlinear evolutions, Pitman Monographs and Surveys in Pure and Applied Mathematics, \#32, Longman Sci. Tech., 1987.

[32] S. Yamamuro, On orthogonally decomposable ordered Banach spaces, Bull. Austral. Math. Soc. 30 (1984) 357-380.

Laboratoire de Mathématiques Appliquées UPRES-A 5033 CNRS

Université de PAU ET DES PAYs de L'Adour

AV. DE L'UNIVERSITÉ 64000

PAU, FRANCE

E-mail address: jean-paul.penot@univ-pau.fr 


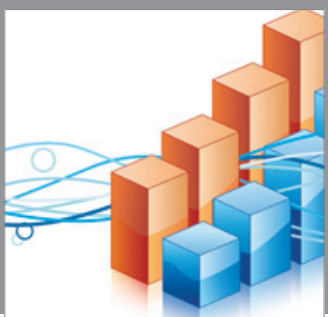

Advances in

Operations Research

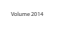

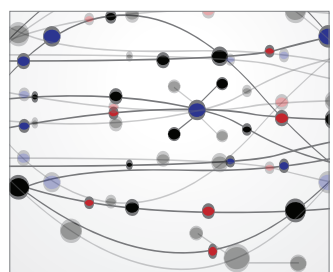

\section{The Scientific} World Journal
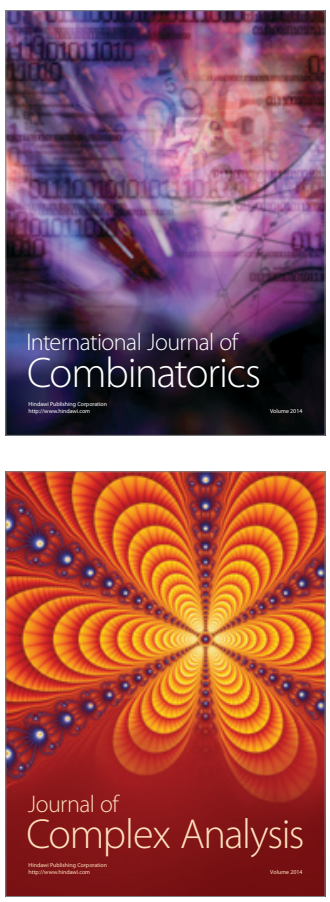

International Journal of

Mathematics and

Mathematical

Sciences
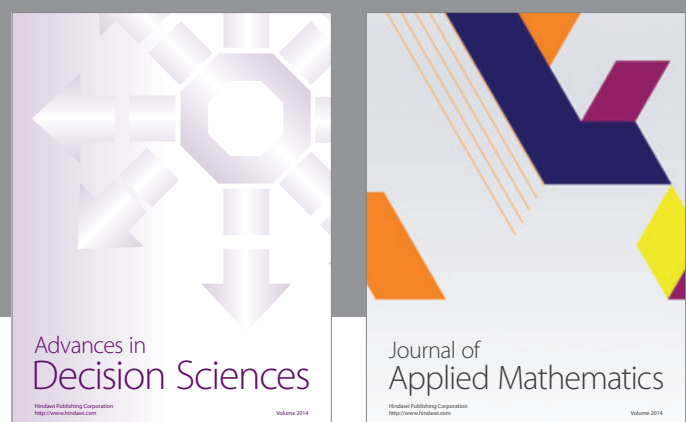

Journal of

Applied Mathematics
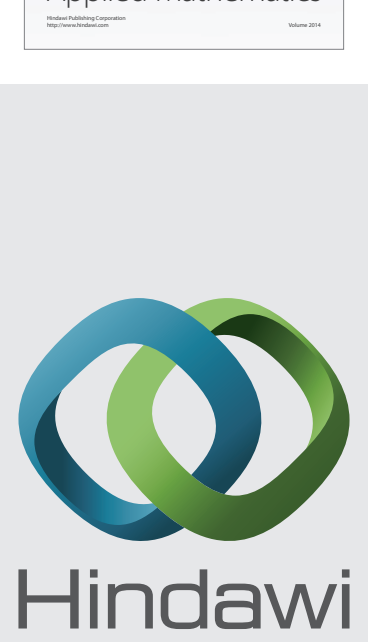

Submit your manuscripts at http://www.hindawi.com
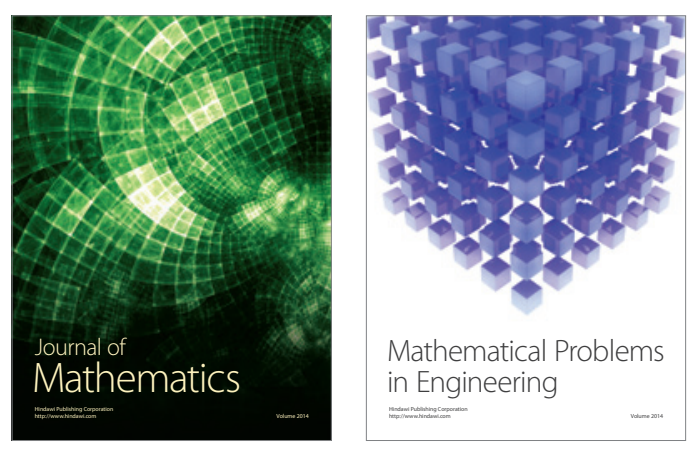

Mathematical Problems in Engineering
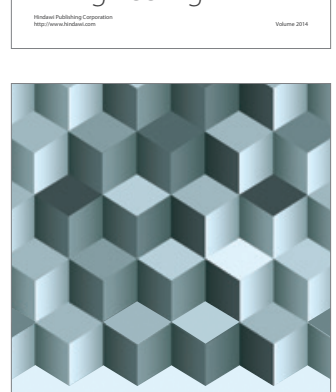

Journal of

Function Spaces
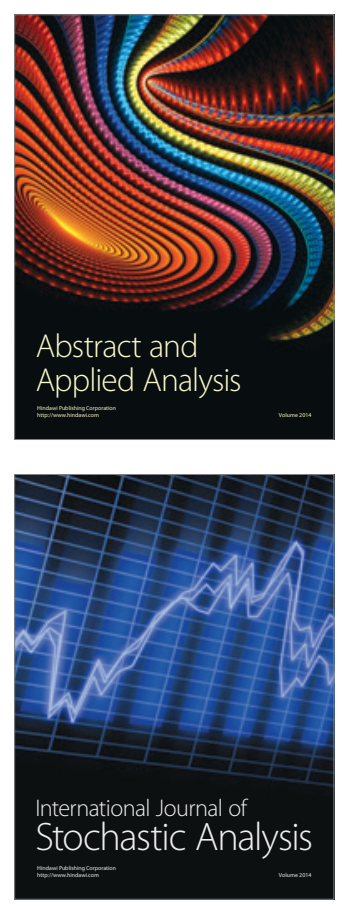

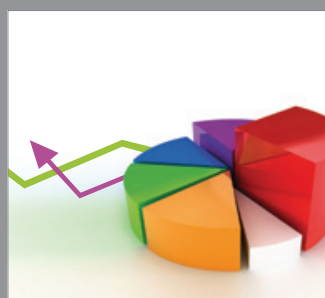

ournal of

Probability and Statistics

Promensencen
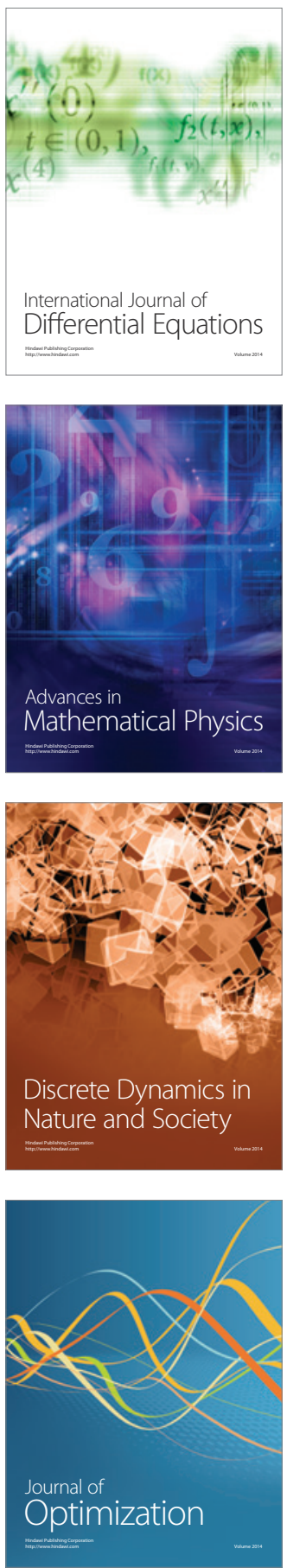